\title{
Feeding of waste milk to Holstein calves affects antimicrobial resistance of Escherichia coli and Pasteurella multocida isolated from fecal and nasal swabs
}

\author{
G. Maynou, ${ }^{*}$ A. Bach, ${ }^{*} \dagger$ and M. Terré ${ }^{* 1}$ \\ *Department of Ruminant Production, Institut de Recerca i Tecnologia Agroalimentàries, 08140 Caldes de Montbui, Spain \\ †Institució Catalana de Recerca i Estudis Avançats, 08010 Barcelona, Spain
}

\begin{abstract}
The use of milk containing antimicrobial residues in calf feeding programs has been shown to select for resistant fecal Escherichia coli in dairy calves. However, information is scarce about the effects of feeding calves waste milk (WM) on the prevalence of multidrugresistant bacteria. The objective of this study was to determine the antimicrobial resistance patterns of fecal E. coli and nasal Pasteurella multocida isolates from calves fed either milk replacer (MR) or WM in 8 commercial dairy farms (4 farms per feeding program). Fecal and nasal swabs were collected from $20 \pm 5$ dairy calves at $42 \pm 3.2 \mathrm{~d}$ of age, and from 10 of these at approximately $1 \mathrm{yr}$ of age in each study farm to isolate the targeted bacteria. Furthermore, resistance of $E$. coli isolates from calf-environment and from 5 calves at birth and their dams was also evaluated in each study farm. Resistances were tested against the following antimicrobial agents: amoxicillin-clavulanic acid, ceftiofur, colistin, doxycycline (DO), enrofloxacin (ENR), erythromycin, florfenicol, imipenem, and streptomycin. A greater number of fecal E. coli resistant to ENR, florfenicol, and streptomycin and more multidrug-resistant $E$. coli phenotypes were isolated in feces of calves fed WM than in those fed MR. However, the prevalence of fecal-resistant E. coli was also influenced by calf age, as it increased from birth to 6 wk of age for ENR and $\mathrm{DO}$ and decreased from $6 \mathrm{wk}$ to 1 yr of age for DO regardless of the feeding program. From nasal samples, an increase in the prevalence of colistin-resistant $P$. multocida was observed in calves fed WM compared with those fed MR. The resistance patterns of E. coli isolates from calves and their dams tended to differ, whereas similar resistance profiles among E. coli isolates from farm environment and calves were observed. The findings of this study suggest that feeding calves
\end{abstract}

Received August 18, 2016.

Accepted December 28, 2016.

${ }^{1}$ Corresponding author: marta.terre@irta.cat
WM fosters the presence of resistant bacteria in the lower gut and respiratory tracts of dairy calves.

Key words: antimicrobial resistance, dairy calf, waste milk

\section{INTRODUCTION}

The use of antimicrobials in animal production constitutes an important issue on the development of resistant bacteria, and it can potentially spread to humans by direct animal contact, food, or the environment (Wegener, 2003). In the dairy industry, antimicrobial agents are used for the treatment of various diseases such as mastitis, metritis, and dry cow therapy, resulting in antimicrobial residues in milk (Bilandžić et al., 2011; De Briyne et al., 2014; Pereira et al., 2014b). To ensure consumer health, milk produced by cows treated with antimicrobials is withdrawn from the dairy industry during both the treatment and milk withdrawal periods (Duse et al., 2013). However, to mitigate milk economic losses, this waste milk (WM) is often used by producers to feed dairy calves (Brunton et al., 2012; Duse et al., 2013).

Although antimicrobial levels in WM could be very low and variable depending on the number of treated cows in the farm and the type of treatment applied, feeding WM to preweaning calves could exert a selective pressure favoring the emergence and dissemination of resistant bacteria in the gut microbiota of calves. It is widely accepted that the selection of antimicrobial resistance occurs when bacterial populations are exposed to antimicrobial agents above the minimal inhibitory concentrations of the susceptible isolates, and the selection of resistant bacteria at extremely low antibiotic concentrations has also been reported (Gullberg et al., 2011). In a recent study, Pereira et al. (2014b) collected WM samples from several dairy farms to screen antimicrobial drug residues, and they found antimicrobial residues below the minimal inhibitory concentration breakpoint for susceptible bacteria in the Enterobacteriaceae family. Later, Pereira et al. (2014a) 
fed calves raw milk with added antimicrobial residues at similar concentrations to those found previously in WM (Pereira et al., 2014b), and they demonstrated an increase in the prevalence of antimicrobial resistance in fecal Escherichia coli isolates.

The consequences of daily WM intake on the development of resistant bacteria in the gut microbiota of calves have been analyzed in several studies obtaining inconsistent results. Whereas Wray et al. (1990) did not observe differences in the antimicrobial resistance of fecal E. coli isolated from calves fed either WM or milk replacer (MR), Aust et al. (2013) reported greater prevalence of resistant fecal E. coli in calves fed unpasteurized and pasteurized WM compared with those fed unprocessed bulk tank milk. However, the number of animals sampled to evaluate the effect of feeding WM on antimicrobial resistance of fecal E. coli was small. Similarly, Langford et al. (2003) observed a positive relationship between the concentrations of penicillin $\mathrm{G}$ in milk fed to calves and the levels of resistance in the lower gut bacterial populations.

Studies evaluating antimicrobial resistance of gut microbiota of calves fed WM have specifically focused on bacterial populations from the gastrointestinal tract (Wray et al., 1990; Langford et al., 2003; Aust et al., 2013), but antimicrobials ingested through milk could also have an effect to other parts of the body, such as respiratory tract, either by direct contact when drinking or by its absorption in the intestine and later spread in the body. For instance, $\beta$-lactam antimicrobials, both penicillins and cephalosporins, are veterinary drugs commonly used in dairy cows (Kerr and Wellnitz, 2003; De Briyne et al., 2014), and penicillins, especially amoxicillin, are antimicrobial agents extremely wellabsorbed in the intestine (Dantzig, 1997; Levison and Levison, 2009). The hypothesis of the present study was that feeding WM to calves will increase the prevalence of gut-resistant bacteria, but it also may foster an increase in the incidence of resistant bacteria in the respiratory tract. Thus, the aim of this study was to determine whether there was an increase of antimicrobial resistance patterns to antimicrobials commonly used in dairy farms in E. coli from fecal samples and in Pasteurella multocida from nasal samples of dairy calves fed WM compared with those fed MR.

\section{MATERIALS AND METHODS}

\section{Dairy Farms, Experimental Treatments, and Sample Collection}

Data were collected from 8 commercial Holstein dairy farms selected out of 69 farms, for which the calf management was known. These farms were located in the region of Girona (Spain) and sampled from December 2013 to September 2015. Farms were selected according to herd size and the milk source that dairy producers used to feed their calves. The average number of milking cows in the farms was 117 with a minimum of 70 and a maximum of 230 . In 4 of the farms, calves were offered MR, and in the other 4 farms, calves were offered WM from cows either receiving antimicrobial therapy or having with high SCC. Calf management practices differed among the study farms and are described in Table 1. Calves were removed from their dams at birth and offered at least 2 feedings of 2 to 3 $\mathrm{L}$ of colostrum. Calves received milk for at least until 6 wk after birth and in some farms calves consumed milk for as long as $12 \mathrm{wk}$ of age. Calves were offered $2 \mathrm{~L}$ of milk (MR or WM) twice daily in most of the farms. Generally, calves were housed individually during the first weeks of life, and then moved to group pens before weaning. The antimicrobial agents used on dairy operations, both to treat or prevent diseases, were recorded from each study farm (Table 2). The most common antimicrobials used to treat or prevent mastitis, either during dry or lactating periods, were $\beta$-lactams (cephalosporin or not), mainly combined with aminoglycosides. The antimicrobial therapy of infectious diseases such as pneumonia or diarrhea in calves, consisted of a $\beta$-lactam (cephalosporin or not) and fluoroquinolone antimicrobial administrated parentally.

In each farm, fecal and nasal swabs were sampled from $20 \pm 5$ female calves at $42 \pm 3.2 \mathrm{~d}$ of age that were enrolled in the study by birth order. The samples were obtained with a sterile transport swab by inserting it into the nasal or rectal cavity and rotating $360^{\circ}$ several times. After sampling, each swab was put into a tub with Amies agar gel (VWR International, Llinars del Vallès, Spain) and kept refrigerated during transportation to the laboratory within the following $4 \mathrm{~h}$. Calves that received antimicrobial therapy before $42 \mathrm{~d}$ of age were excluded from the study. Following the same sampling procedure, $10 \pm 3$ calves per farm that were previously sampled and never received antimicrobial therapy were sampled again at $1.3 \pm 0.16 \mathrm{yr}$ of age. These samples were obtained from July 2015 to September 2015, and those heifers in the farms that were about 1 yr old at that time were selected.

To evaluate the possibility of acquisition antimicrobial-resistant bacteria from other sources, such as the dam at calving or the farm environment, from June 2014 to June 2015 fecal swabs from $5 \pm 1$ randomly selected calves were collected at birth and at $6 \mathrm{wk}$ of age and also a fecal swab was obtained from their respective dams after calving. Environmental samples were collected during February and March 2015 once per farm from the calving area $(\mathbf{C A})$, calf-housing area 
(HA), and calf-feeder area (FA; Table 3$)$. For the CA sampling, 5 randomly selected sites on the floor surface were swabbed using a sterile flocked swab from a transport tube (Puritan Medical Products LLC, Guilford, ME) moistened in situ with nutrient broth $(0.5 \mathrm{~g} / \mathrm{L}$ of beef extract, $1 \mathrm{~g} / \mathrm{L}$ of peptone, and 0.5 $\mathrm{g} / \mathrm{L}$ of $\mathrm{NaCl}$ ). The HA was sampled following the same procedure for the walls and floor. When calves were housed individually, samples were collected from 5 pens randomly selected using the same swab moistened only once with nutrient broth. Two randomly selected calf hutches were sampled when calves were housed in groups. Samples from FA were collected by swabbing first the water bowls and then the feeding troughs using a dry swab. For individual calf pens, the water and feeding buckets of the 5 selected pens were sampled with the same swab, whereas for grouped calf hutches, only 3 randomly selected water buckets were swabbed along with feeding troughs. For each farm, 3 buckets or feeding bottles used to offer milk to the calves were also swabbed using a dry swab. All environmental samples were maintained on ice until samples were processed at the laboratory for bacteriological culture.

\section{Bacteria Isolation, Culture, and Identification by PCR}

Fecal and environment swabs were streaked onto Agar McConkey (Oxoid, Madrid, Spain) and incubated for $24 \mathrm{~h}$ at $37^{\circ} \mathrm{C}$. Then, 5 fecal suspected E. coli colonies were isolated from fecal swabs and 3 from the environmental swabs. Nasal swabs were cultured, following the same procedure and under the same conditions as fecal swabs, on Columbia agar with 5\% sheep blood (Oxoid) for isolation of 5 suspected P. multocida colonies. Suspected colonies were selected by morphologic characteristics after incubation, and subcultured for another $24 \mathrm{~h}$ on Columbia agar with $5 \%$ sheep blood to obtain pure bacterial cultures.
For isolate identification, E. coli were subjected to specific PCR assays using a set of primers reported by Juck et al. (1996): forward (5'-ATCACCGTGGTGACGCATGTCGC-3') and reverse (5'-CACCACGATGCCATGTTCATCTGC-3'; Integrated DNA Technologies, Leuven, Belgium), resulting in a fragment of $486 \mathrm{bp}$. The primers set reported by Townsend et al. (1998) were used to identify P. multocida: KMT1SP6 (5'-GCTGTAAACGAACTCGCCAC-3') and KMT1T7 (5'-ATCCGCTATTTACCCAGTGG-3'; Integrated DNA Technologies), obtaining a product of approximately 460 bp. For DNA amplification, each bacterial pure culture was inoculated into $20 \mu \mathrm{L}$ of Milli-Q water, and $2 \mu \mathrm{L}$ of these suspensions was added directly in $23 \mu \mathrm{L}$ of reaction mixture. Reaction mixtures contained $1 \times$ Taq buffer with $\mathrm{Mg}^{2+}, 200 \mu M$ of deoxynucleotide triphosphates, $0.5 \mathrm{U}$ of Taq DNA polymerase (Biorbyt, Cambridge, UK), and $0.125 \mu M$ of each primer. The PCR reactions were conducted using a thermocycler iCycler (Bio-Rad, Hercules, CA) and cycled as follows: an initial denaturing step at $95^{\circ} \mathrm{C}$ for $5 \mathrm{~min}$, followed by 30 cycles at $95^{\circ} \mathrm{C}$ for 1 min, $55^{\circ} \mathrm{C}$ for $1 \mathrm{~min}$, and $72^{\circ} \mathrm{C}$ for $1 \mathrm{~min}$, and a final extension of $10 \mathrm{~min}$ at $72^{\circ} \mathrm{C}$. All PCR were run with positive controls using E. coli ATCC 25922 and $P$. multocida CECT 962, and negative controls containing Milli-Q water in place of the bacterial suspensions. The presence of PCR products was confirmed by electrophoresis on $1.5 \%$ agarose gels with SYBR safe DNA gel stain (Thermo Fisher Scientific SL, Barcelona, Spain) in $1 \times$ Tris-acetate-EDTA running buffer (Merck KGaA, Darmstadt, Germany) and visualized under UV light. After bacteria identification, each confirmed colony was picked from bacterial pure culture to a $1.5-\mathrm{mL}$ tube containing $334 \mu \mathrm{L}$ of brain heart infusion broth (Scharlab SL, Sentmenat, Spain) and $666 \mu \mathrm{L}$ of $30 \%$ glycerol (Panreac Química SLU, Castellar del Vallès, Spain), and frozen at $-80^{\circ} \mathrm{C}$.

Table 1. Milk regimen and calf-housing system in each study farm

\begin{tabular}{lccccc}
\hline & \multicolumn{2}{c}{ Calf feeding program } & & \multicolumn{2}{c}{ Calf-housing system } \\
\cline { 2 - 3 } \cline { 5 - 6 } Farm & $\begin{array}{c}\text { Duration of preweaning } \\
\text { period, wh }\end{array}$ & $\begin{array}{c}\text { Milk offered, } \\
\text { L/feeding }\end{array}$ & & $\begin{array}{c}\text { Individual } \\
\text { housing, } d\end{array}$ & $\begin{array}{c}\text { Number of } \\
\text { calves/pen }\end{array}$ \\
\hline 1 & 8 & 2.0 & & 30 & $5-6$ \\
2 & 13 & 2.0 & & 42 & $4-5$ \\
3 & 10 & 2.0 & & 30 & 6 \\
4 & 8 & 1.75 & & 14 & 5 or 15 \\
5 & 6 & 2.0 & & 42 & $2-3$ \\
6 & 8 & 2.0 & & 0 & 6 \\
7 & 6 & 2.0 & & 21 & 5 or 10 \\
8 & 12 & 2.75 & & &
\end{tabular}




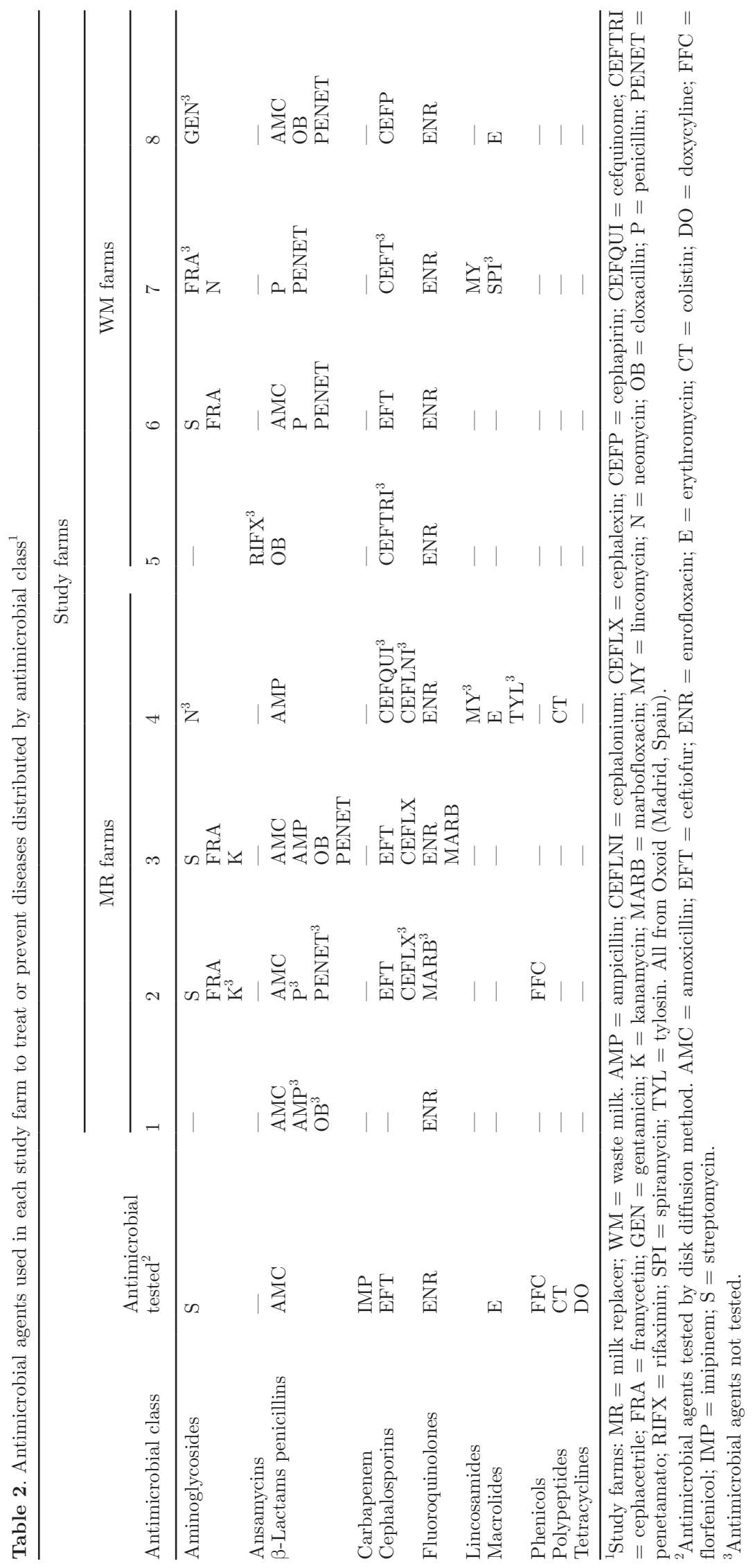




\section{Antimicrobial Susceptibility Test}

The susceptibility of the confirmed isolates against antimicrobial agents was determined by the disk diffusion method described by the National Committee for Clinical Laboratory Standards with some modifications of the procedure (CLSI, 2002). Each isolated bacteria from pure culture was suspended into $2 \mathrm{~mL}$ of $0.9 \% \mathrm{NaCl}$ sterile solution and adjusted spectrophotometrically between 0.08 to 0.10 of absorbance at $625 \mathrm{~nm}$ (equivalent to $0.5 \mathrm{McFarland}$ standard). After adjusting for turbidity, the sterile cotton swab was dipped in the E. coli suspension and then rolled over the dry surface of Mueller-Hinton agar plates (Oxoid), covering it evenly to obtain uniform growth. Mueller-Hinton agar with 5\% sheep blood plates (Oxoid) was inoculated following the same procedure with dipped cotton swabs into $P$. multocida suspension. The tested antimicrobial disks were placed on the surface of each agar plates using a disk dispenser (Oxoid). The antimicrobial agents tested were amoxicillinclavulanic acid (AMC), 20/10 $\mu \mathrm{g}$; ceftiofur (EFT), $30 \mu \mathrm{g}$; colistin (CT), $10 \mu \mathrm{g}$; doxycycline (DO), 30 $\mu \mathrm{g}$; enrofloxacin (ENR), $5 \mu \mathrm{g}$; erythromycin (E), 15 $\mu \mathrm{g}$; florfenicol (FFC), $30 \mu \mathrm{g}$; imipenem (IMP), 10 $\mu \mathrm{g}$; and streptomycin (S), $10 \mu \mathrm{g}$ (Oxoid). The plates were incubated aerobically between 16 and $18 \mathrm{~h}$ at $37^{\circ} \mathrm{C}$ for E. coli susceptibility test and 20 to $24 \mathrm{~h}$ for $P$. multocida plates under the same conditions as $E$. coli. After incubation, the zones of inhibition around each disk were measured in millimeters. Obtained values were then classified into 3 categories (resistant, sensitive, or intermediate) following the interpretative criteria adhering to the Clinical and Laboratory Standards Institute (CLSI) guidelines as previously described (Benedict et al., 2015; Kar et al., 2015). Because no CLSI breakpoints were available for CT, the interpretative criteria described by Galani et al. (2008) for susceptibility testing in Enterobacteriaceae were used. Breakpoints used to categorize antimicrobial susceptibility of isolates are specified in Table 4. Internal quality control was performed using $E$. coli ATCC 25922 as a reference strain for each batch of isolates tested.

\section{Statistical Analyses}

To evaluate the effect of feeding WM or MR on fecal E. coli and nasal P. multocida resistance to antimicrobial agents tested in calves at 6 wk of age, 2 statistical analyses were performed:

(1) A general linear mixed model using the PROC GLIMMIX procedure of SAS (version 9.2, SAS Institute Inc., Cary, NC), in which the proportion of resistant bacteria isolated per calf and for each antimicrobial tested was considered as the dependent variable. Milk feeding practice (MR or WM) was the fixed effect of the model, and farm was considered the random effect. The use within the farm of any of the antimicrobial class tested was used as a block in the statistical model.

(2) Based on bacteria-resistant profiles represented by the size of the inhibition zones to each of the 9 antimicrobials tested, multidrug resistance phenotypes among E. coli and P. multocida isolates were described independently by cluster analyses using the Ward's minimum variance method of the PROC CLUSTER procedure. The final number of clusters was determined using the pseudo F statistic and pseudo- $\mathrm{T}^{2}$ statistic as described by Yoder et al. (2014). A frequency analysis of the cluster distribution between bacteria isolated

Table 3. Schedule of the different environmental areas sampled for each of the milk replacer and waste milk farms according to their calf-housing system

\begin{tabular}{|c|c|c|c|c|c|c|}
\hline \multirow[b]{3}{*}{ Farm $^{1}$} & \multirow[b]{3}{*}{$\mathrm{CA}$} & \multicolumn{4}{|c|}{ Sampling areas ${ }^{2}$} & \\
\hline & & \multicolumn{2}{|c|}{ HA } & \multicolumn{3}{|c|}{ FA } \\
\hline & & IP & GP & IP & GP & MB \\
\hline 1 & $\mathrm{X}$ & $\mathrm{X}$ & $\mathrm{X}$ & X & X & $\mathrm{X}$ \\
\hline 2 & $\mathrm{X}$ & $\mathrm{X}$ & - & $\mathrm{X}$ & - & $\mathrm{X}$ \\
\hline 3 & $\mathrm{X}$ & $\mathrm{X}$ & $\mathrm{X}$ & $\mathrm{X}$ & $\mathrm{X}$ & $\mathrm{X}$ \\
\hline 4 & $X$ & $X$ & $X$ & $X$ & $X$ & $X$ \\
\hline 5 & $\mathrm{X}$ & $\mathrm{X}$ & - & $\mathrm{X}$ & - & $\mathrm{X}$ \\
\hline 6 & $\mathrm{X}$ & - & $\mathrm{X}$ & - & $X$ & X \\
\hline 7 & $\mathrm{X}$ & $\mathrm{X}$ & - & $\mathrm{X}$ & - & $\mathrm{X}$ \\
\hline 8 & $\mathrm{X}$ & $\mathrm{X}$ & $\mathrm{X}$ & $\mathrm{X}$ & $X$ & $X$ \\
\hline
\end{tabular}

${ }^{1}$ In farms 1 to 4 , calves were fed milk replacer, and in farms 5 to 8 , calves were fed waste milk.

${ }^{2}$ Environmental sampling areas: $\mathrm{CA}=$ calving area; $\mathrm{HA}=$ housing area; $\mathrm{FA}=$ feeding area; $\mathrm{IP}=$ individual pen; $\mathrm{GP}=$ group pen; $\mathrm{MB}=$ milk bucket or bottle. 
Table 4. Zone diameters and interpretative criteria of tested antimicrobial agents used to categorize antimicrobial susceptibility of Escherichia coli and Pasteurella multocida isolates

\begin{tabular}{|c|c|c|c|c|c|}
\hline Antimicrobial agents tested & Disk content, $\mu \mathrm{g}$ & Resistant & Intermediate & Susceptible & CLSI guideline $^{1}$ \\
\hline Ceftiofur $^{3}$ & 30 & $\leq 17$ & $18-20$ & $\geq 21$ & VET01-A4, 2013 \\
\hline Enrofloxacin $^{3}$ & 5 & $\leq 16$ & $17-20$ & $\geq 21$ & VET01-A4, 2013 \\
\hline Erythromycin ${ }^{5}$ & 15 & $\leq 13$ & $14-22$ & $\geq 23$ & M100-S24, 2014 \\
\hline Florfenicol $^{3}$ & 30 & $\leq 14$ & $15-18$ & $\geq 19$ & VET01-A4, 2013 \\
\hline
\end{tabular}

${ }^{1}$ CLSI $(2002,2008,2013,2014)$.

${ }^{2}$ Interpretative criteria defined for Enterobacteriaceae applied in both E. coli and P. multocida as CLSI does not define zone diameter breakpoints in Pasteurella spp. for these antimicrobial agents.

${ }^{3}$ Interpretative criteria defined for $P$. multocida in cattle applied in both E. coli and P. multocida as CLSI does not define zone diameter breakpoints in Enterobacteriaceae for these antimicrobial agents.

${ }^{4}$ No zone diameter breakpoints are defined by the CLSI for colistin, and the ones described by Galani et al. (2008) were followed.

${ }^{5}$ Interpretative criteria defined for Enterococcus spp. applied in both E. coli and P. multocida as CLSI does not define zone diameter breakpoints in both Enterobacteriaceae and Pasteurella spp. for these antimicrobial agents.

from calves fed MR and WM was performed by a chisquared test using the PROC FREQ procedure.

Feeding practice (MR or WM) and calf-age ( $0 \mathrm{~d}, 42$ d, or 1 yr) effect on the proportion of resistant fecal $E$. coli isolates were also analyzed using generalized linear mixed models for each antimicrobial tested. Data were split in 2 blocks: from birth to 6 wk of age, and from 6 wk to 1 year of age, considering only the animals that were sampled at both times ( 36 calves at birth and at 6 wk of age, and 69 calves at 6 wk and 1 yr of age). For this analysis, feeding practice, calf age, and its interaction were the fixed effects of the model; farm was the random effect, and the proportion of resistant colonies to each antibiotic tested per calf was the dependent variable. Due to the low isolation of nasal P. multocida at $1 \mathrm{yr}$, this analysis was not performed with data obtained from nasal swabs.

To evaluate the relationship among bacterial antimicrobial resistance in calves, their dams, and their environment, E. coli colonies isolated from the farm environment and cows and their calves sampled at birth and at 6 wk of age were grouped at the farm level. Then, 2 fecal $E$. coli clustering analyses were conducted for each farm: (1) E. coli isolates from cow and their calves at birth, and (2) E. coli isolates from calves (at birth and at 6 wk of age) and their environment (CA, HA, and FA). Then, the most predominant resistant profile among all colonies isolated from each calf, cow, and environmental site was chosen, and they were paired (cow/calf and calf/environment). These data were analyzed by a Wilcoxon signed-rank test using the PROC UNIVARIATE procedure. Finally, antimicrobial resistance clusters were classified as multidrug ( $\geq 3$ antimicrobial resistances) or non-multidrug profiles, and the frequency of these profiles among cows and calves at birth were analyzed with a chi-squared test.

\section{RESULTS}

\section{Descriptive Data}

A total of 1,485 E. coli colonies were confirmed from fecal swabs, including 785 of 825 from calves at $6 \mathrm{wk}$ of age, 180 of 180 from calves at birth, 176 of 180 from their dams, and 344 of 345 from calves at 1 yr old. Among all calves and cows sampled, at least $2 \mathrm{E}$. coli colonies were isolated from each fecal swab. From the 825 colonies isolated from nasal swabs of calves at $6 \mathrm{wk}$ of age, only 301 were confirmed as P. multocida, and 5 of 345 colonies from calves at 1 yr of age. Among the 165 nasal swabs of calves at 6 wk of age, only 77 provided $P$. multocida colonies ranging from 1 to 5 colonies per calf, whereas among the 69 calves sampled at $1 \mathrm{yr}$ of age, only 3 provided P. multocida isolates, ranging from 1 to 2 colonies per calf. In total, 40 environmental samples were collected among all the study farms of which 38 provided E. coli colonies ranging from 2 to 3 colonies per sample. The total number of environmental E. coli isolates was 102, including 23 from CA, 37 from $\mathrm{FA}$, and 42 from $\mathrm{HA}$.

\section{Effect of Calf-Feeding Practices on Antimicrobial Resistance}

No isolates of E. coli or P. multocida resistant to IMP were isolated from calves in this study. Results from the general linear mixed-effects model indicated that in both milk feeding practices, 90.7 and $45.3 \%$ of the $E$. 
Table 5. Percentage, odds ratio (OR), and confidence interval of the general linear mixed effects model assessing the effect of feeding calves milk replacer (MR) or waste milk (WM) on the presence of resistant fecal Escherichia coli to 8 antimicrobials

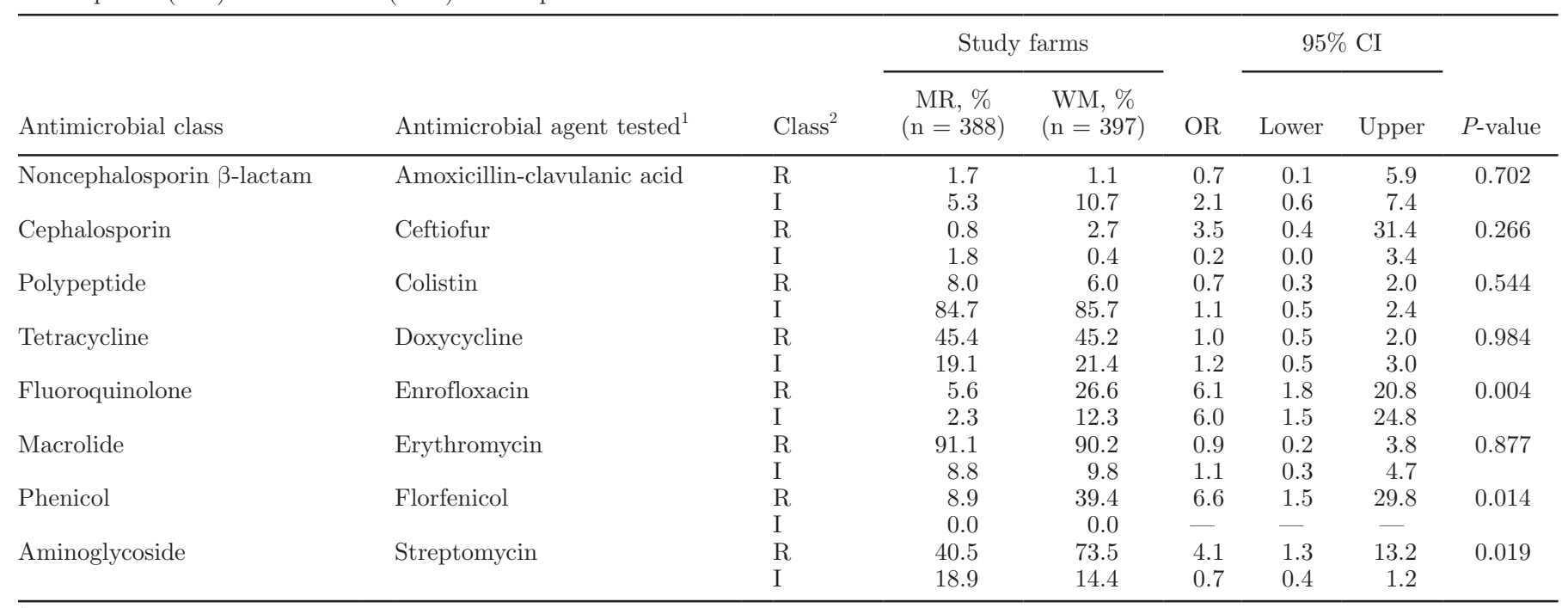

${ }^{1}$ Imipenem was not included in the analysis because no resistant isolates were detected.

${ }^{2} \mathrm{R}=$ resistant; $\mathrm{I}=$ intermediate.

coli isolates were resistant to $\mathrm{E}$ and DO, respectively, and low percentages of resistant E. coli isolates were observed for AMC, EFT, and CT, also being similar in both treatments (Table 5). In contrast, percentages of resistant $E$. coli isolates per calf at 6 wk of age for ENR $(P<0.01)$, FFC $(P<0.05)$, and $\mathrm{S}(P<0.05)$ were greater in WM compared with MR calves (Table 5). The antimicrobial agents used in the study farms to treat or prevent diseases (used as a block in the statistical model) did not have any effect on the proportion of resistant $E$. coli per calf for any of the antimicrobial agents tested. According to the pseudo $\mathrm{F}$ and pseudo- $\mathrm{T}^{2}$ statistics of PROC CLUSTER, the level of clustering was determined and 5 antimicrobial-resistance clusters were created to describe the antimicrobial-resistance profiles in the present study (Table 6). Calves fed WM presented a greater $(P<0.05)$ percentage of $E$. coli isolates exhibiting multidrug resistance profiles $(\geq 3$ antimicrobial agents, clusters D, E) compared with MR-fed calves (Table 6). In contrast, greater $(P<$ 0.01 ) percentages of $E$. coli colonies resistant to 1 or 2 antimicrobial agents (clusters A, B) were observed in MR-fed compared with WM-fed calves (Table 6).

Pasteurella multocida isolates from nasal swabs presented only resistance to CT (24.7\% of isolates), E (5.5\% of isolates), and S (77.7\% of isolates) among the 9 antimicrobials tested. Calves fed WM had a greater proportion of resistant bacteria to $\mathrm{CT}(P=0.05)$ than

Table 6. Cluster distribution of Escherichia coli isolated from calves at 6 wk of age fed milk replacer (MR) or waste milk (WM) during the preweaning period

\begin{tabular}{|c|c|c|c|c|}
\hline \multirow[b]{2}{*}{ Cluster } & \multirow[b]{2}{*}{ Antimicrobial-resistant phenotypes ${ }^{1}$} & \multicolumn{2}{|c|}{ Resistant isolates, ${ }^{2} \%$} & \multirow[b]{2}{*}{$P$-value } \\
\hline & & $\begin{array}{c}\mathrm{MR} \\
(\mathrm{n}=388)\end{array}$ & $\begin{array}{c}\text { WM } \\
(\mathrm{n}=397)\end{array}$ & \\
\hline A & $\mathrm{E}-\mathrm{CT}^{33}$ & 25.5 & 19.6 & $<0.001$ \\
\hline $\mathrm{B}$ & $\mathrm{DO}-\mathrm{E}-\mathrm{CT}^{3}$ & 30.1 & 11.3 & $<0.001$ \\
\hline $\mathrm{C}$ & $\mathrm{E}-\mathrm{S}-\mathrm{DO}^{4}-\mathrm{CT}^{3}$ & 66.0 & 33.0 & 0.548 \\
\hline $\mathrm{D}$ & $\mathrm{DO}-\mathrm{ENR}-\mathrm{E}-\mathrm{S}-\mathrm{CT}^{3}$ & 3.9 & 19.1 & $<0.001$ \\
\hline $\mathrm{E}$ & $\mathrm{DO}-\mathrm{E}-\mathrm{FFC}-\mathrm{S}-\mathrm{CT}^{3}-\mathrm{ENR}^{, 5}$ & 8.5 & 19.9 & 0.049 \\
\hline \multicolumn{5}{|c|}{$\begin{array}{l}{ }_{1}^{1} \mathrm{CT}=\text { colistin; } \mathrm{DO}=\text { doxycycline; } \mathrm{ENR}=\text { enrofloxacin } ; \mathrm{E}=\text { erythromycin; } \mathrm{FFC}=\text { florfenicol } ; \mathrm{S}=\text { streptom } \\
\text { cin. Apostrophes denote intermediate level of resistance. }\end{array}$} \\
\hline \multicolumn{5}{|c|}{${ }^{2}$ Percentages of resistant isolates by feeding treatment. } \\
\hline \multicolumn{5}{|c|}{$\begin{array}{l}{ }^{3} \text { Intermediate level of resistance to CT according to Clinical and Laboratory Standards Institute (CLS } \\
\text { breakpoints. }\end{array}$} \\
\hline \multicolumn{5}{|c|}{${ }^{4}$ Intermediate level of resistance to DO according to CLSI breakpoints. } \\
\hline
\end{tabular}


Table 7. Cluster distribution of Pasteurella multocida isolated from calves at 6 wk of age fed milk replacer (MR) or waste milk (WM) during the preweaning period

\begin{tabular}{|c|c|c|c|c|}
\hline \multirow[b]{2}{*}{ Cluster } & \multirow{2}{*}{$\begin{array}{l}\text { Antimicrobial-resistant } \\
\text { phenotypes }^{1}\end{array}$} & \multicolumn{2}{|c|}{ Resistant isolates, ${ }^{2} \%$} & \multirow[b]{2}{*}{$P$-value } \\
\hline & & $\mathrm{MR}(\mathrm{n}=194)$ & $\mathrm{WM}(\mathrm{n}=107)$ & \\
\hline A & - & 6.2 & 25.2 & $<0.001$ \\
\hline B & $S-E^{, 3}$ & 62.4 & 0.9 & $<0.001$ \\
\hline $\mathrm{C}$ & $\mathrm{CT}-\mathrm{E}^{, 3}-\mathrm{S}^{, 4}$ & 4.1 & 73.0 & $<0.001$ \\
\hline $\mathrm{D}$ & $\mathrm{E}-\mathrm{S}$ & 27.3 & 0.9 & $<0.001$ \\
\hline
\end{tabular}

${ }^{1} \mathrm{CT}=$ colistin; $\mathrm{E}=$ erythromycin; $\mathrm{S}=$ streptomycin. Apostrophes denote intermediate level of resistance.

${ }^{2}$ Percentages of resistant isolates by feeding treatment. $\mathrm{MR}=$ milk replacer; $\mathrm{WM}=$ waste milk.

${ }^{3}$ Intermediate level of resistance to E according to Clinical and Laboratory Standards Institute (CLSI) breakpoints.

${ }^{4}$ Intermediate level of resistance to S according to CLSI breakpoints.

MR-fed calves. Similar to fecal E. coli isolates, the antimicrobial agents used in study farms did not have any effect on the prevalence of resistant $P$. multocida for any of the antimicrobial agents tested. However the cluster analysis indicated that more $(P<0.001)$ calves fed WM had a non-antimicrobial resistance pattern compared with MR-fed calves, and WM calves presented more CT (cluster C) and less S and E (cluster $\mathrm{D} ; P<0.001)$ resistance patterns than MR-fed calves (Table 7).

\section{Effect of Calf Feeding Practices and Calf Age on Antimicrobial Resistance}

No differences in the incidence of $E$. coli resistant to AMC, EFT, CT, DO, FFC, and E were found in the isolates from birth to 6 wk of age. In contrast, the proportion of resistant $E$. coli to ENR increased $(P<$ 0.05 ) with calf age (from 0.003 to $0.16 \pm 0.057$ ), and decreased for $\mathrm{S}(P<0.05$; from 0.63 to $0.31 \pm 0.258)$ (Figure 1). The evolution of the proportion of E. coli resistant to FFC $(P<0.05)$ and $\mathrm{S}(P<0.05)$ from birth to $6 \mathrm{wk}$ of age was different depending on the milk feeding practices. The proportion of E. coli resistant to FFC increased $(P<0.05)$ with age in calves fed MR (from 0.73 to $0.98 \pm 0.883$ ). In contrast, WM-fed calves maintained high proportions of E. coli resistant to FFC from birth to 6 wk of age $(0.94 \pm 0.723$ and $0.88 \pm$ 0.664, respectively). Contrary to FFC, MR-fed calves maintained similar proportions of $E$. coli resistant to $\mathrm{S}$ (from 0.62 to $0.49 \pm 0.352$ ), and WM-fed calves had a decrease $(P<0.05)$ in the proportion of resistant $E$. coli from birth to $6 \mathrm{wk}$ of age (from 0.64 to $0.17 \pm$ 0.376).

The proportion of E. coli resistant to AMC, EFT, and CT in calves sampled at both 6 wk and 1 yr of age was in the range of 0 to $5 \%$ of the total E. coli isolates, and no differences in $E$. coli resistant to these antimicrobials were found from 6 wk to $1 \mathrm{yr}$ of age.
The proportion of E. coli resistant to DO and ENR decreased $(P<0.05)$ and those of $\mathrm{E}, \mathrm{FFC}$, and $\mathrm{S}$ increased $(P<0.05)$ from 6 wk to 1 yr of age (Figure 2$)$. Calves fed MR tended $(P=0.06)$ to have lower and greater proportions of resistant $E$. coli isolates to $\mathrm{E}$ and $\mathrm{S}(\mathrm{E}=91.1 \%, \mathrm{~S}=90.7 \%)$, respectively, than WM-fed calves $(\mathrm{E}=97.6 \%, \mathrm{~S}=57.0 \%)$ in calves sampled at 6 wk and $1 \mathrm{yr}$ of age. No interaction was present between feeding practices and calf age (6 wk and 1 yr) for any of the antimicrobial agents tested.

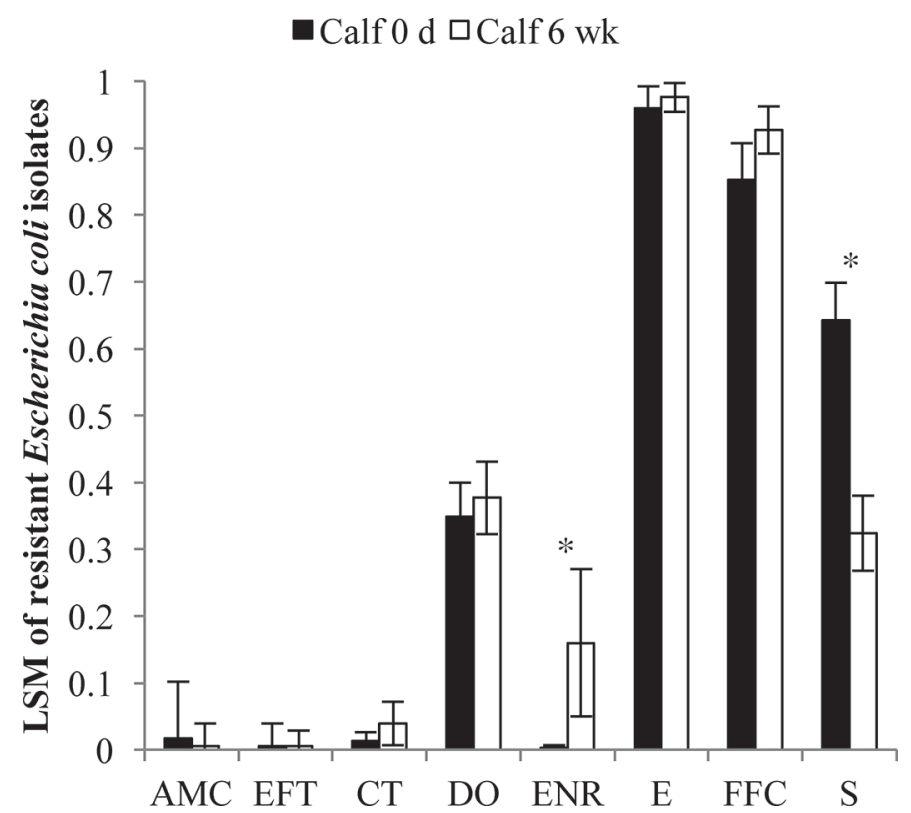

Figure 1. Proportion of fecal Escherichia coli resistant to amoxicillin-clavulanic acid (AMC), ceftiofur (EFT), colistin (CT), doxycycline (DO), enrofloxacin (ENR), erythromycin (E), florfenicol (FFC), and streptomycin (S) isolated from the same dairy calves at birth and at 6 wk of age. Error bars indicate SEM, and asterisks denote differences between feeding programs within antimicrobial type. 
Table 8. Percentage of both non-multidrug- and multidrug-resistant Escherichia coli isolated from fecal swabs of newborn calves and their respective dams

\begin{tabular}{lccc}
\hline & \multicolumn{2}{c}{ Resistant isolates, $\%$} & \\
\cline { 2 - 3 } Item & Cow $(\mathrm{n}=176)$ & Calf $(\mathrm{n}=180)$ & $P$-value ${ }^{1}$ \\
\hline Non-multidrug-resistant profile & 92.6 & 80.6 & $<0.0009$ \\
Multidrug-resistant profile & 7.4 & 19.4 & $<0.0009$ \\
\hline
\end{tabular}

${ }^{1} P$-value obtained by chi-squared test.

\section{Relationship Between Bacterial Antimicrobial Resistances}

Calf and Its Dam. The main antimicrobial-resistant phenotype of E. coli isolated from calves and their dams tended $(P=0.09)$ to be different. The frequency of multidrug-resistant profiles in isolates from calves at birth was greater compared with that of their dams (Table 8). Forty-eight multidrug-resistant E. coli were isolated from cows and calves at birth, from which $81.3 \%$ exhibited resistance to at least DO, E, and S. In the non-multidrug-resistant clusters, E. coli colonies resistant to E were present in $92.5 \%$ of the colonies.

Calf and Environment. No differences were observed between paired resistant clusters of calves at birth and CA. Similarly, no differences were observed between paired resistant clusters of calves at 6 wk of age

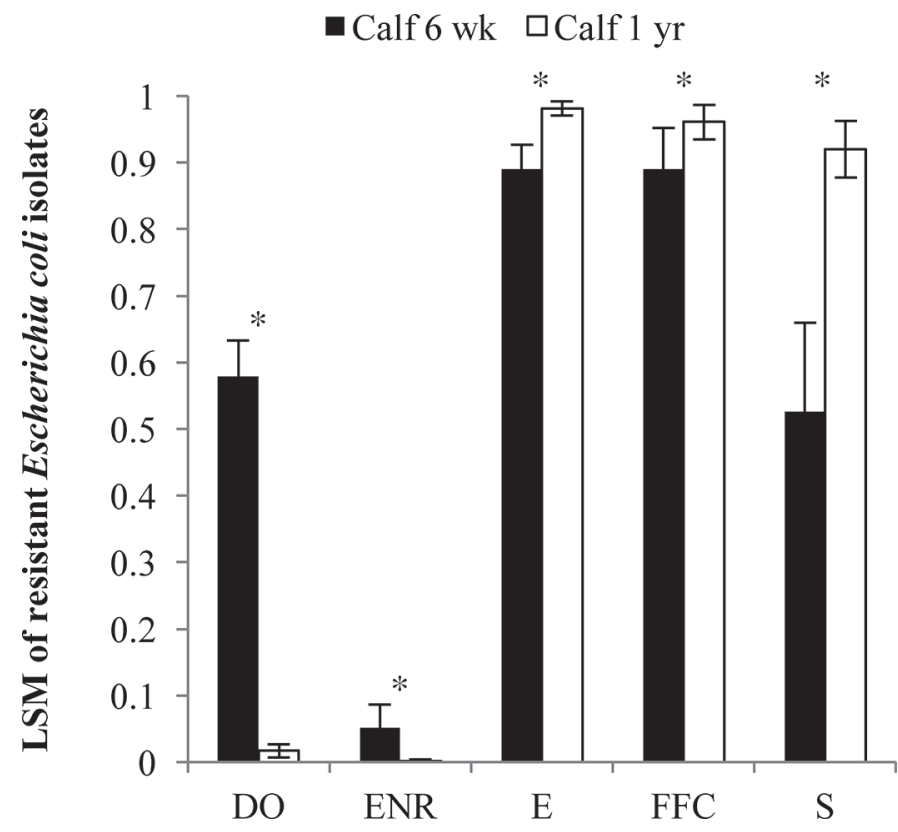

Figure 2. Proportion of fecal Escherichia coli resistant to doxycycline (DO), enrofloxacin (ENR), erythromycin (E), florfenicol (FFC), and streptomycin $(\mathrm{S})$, isolated from the same calves at $6 \mathrm{wk}$ (during preweaning period) and at 1 yr of age. Error bars indicate SEM, and asterisks denote differences between feeding programs within antimicrobial type. and their HA and FA. Four hundred sixty-two E. coli were isolated from the environment and calves at birth and at 6 wk of age, from which $43.4 \%$ exhibited single resistance to $\mathrm{E}$, and $17.9 \%$ exhibited a resistance to both DO and E. The most common multidrug-resistant phenotype (13.6\%) observed in E. coli isolates from the environment and calves at birth and at 6 wk of age was against ENR, E, and S.

\section{DISCUSSION}

In the present study, WM was not subjected to antimicrobial residues analysis. However, greater increases in the prevalence of resistant fecal E. coli were observed for ENR, FFC, and S in calves fed WM than in those fed MR. Two of these antimicrobials were commonly used in the study farms (ENR, for the treatment of digestive and respiratory infections in calves, and S, for the treatment of IMI in lactating cows) (Table 2), whereas FFC was only used in one of the MR farms for the treatment of respiratory diseases in both calves and cows, and the isolation of $E$. coli resistant to this antimicrobial was not expected. Similarly, Berge et al. (2006) reported that feeding calves a MR containing neomycin sulfate and tetracycline $\mathrm{HCl}$ selected fecal $E$. coli resistant to aminoglycosides other than neomycin, chloramphenicol, and sulfonamides, which were not included in the diet of the calves. Gow et al. (2008) observed associations among different resistant genes in fecal E. coli isolates in cattle, suggesting that the use of a particular antimicrobial could select for resistance to other antimicrobials within a bacterial population. In particular, resistance to FFC in E. coli isolates from cattle was found to be mediated by the gene flo $R$ in several studies (Cloeckaert et al., 2000; White et al., 2000; Doublet et al., 2002) and this gene has been observed along with other resistant genes in mobile genetic elements, which would increase the risk of co-selection and horizontal dissemination of resistant E. coli in animals (Meunier et al., 2010).

Beta-lactams (whether cephalosporins or not) were also antimicrobial agents commonly used in the study farms (Table 2) to treat or prevent mastitis in lactating cows and for the treatment of pneumonia or diarrhea in 
dairy calves. Hence, high levels of resistance in bacterial isolates were expected. However, low percentages of resistance to AMC and EFT were found in E. coli from calves fed both types of milk, whereas no resistance to these antimicrobials was detected in P. multocida. The most common mechanisms of resistance to $\beta$-lactams are through the expression of different types of $\beta$-lactamases, and in most cases this resistance is acquired by horizontal gene transfer from other bacteria using genetic mobile elements such as conjugative plasmids or transposons (Philippon et al., 2002; Queenan and Bush, 2007; Shaikh et al., 2015). The occurrence of $\beta$-lactam-resistant bacteria has been found to vary greatly among different geographical areas and bacterial species (Winokur et al., 2001; Stürenburg and Mack, 2003; Hendriksen et al., 2008), suggesting that resistant bacteria are not likely to arise by antimicrobial pressure alone.

Interestingly, high levels of resistance to DO and $\mathrm{E}$ were observed among $E$. coli isolates in calves at birth and at $6 \mathrm{wk}$ of age regardless of feeding treatment and these antimicrobials were either not used (DO) or only used in 2 of the 8 study farms (E) to treat respiratory diseases, mastitis, and metritis (Table 2). Several authors (Shoemaker et al., 2001; Yates et al., 2004; Bischoff et al., 2005) have suggested that increases in antimicrobial-resistant enteric bacteria may be linked to an efficient horizontal transfer of resistant genes among different species of bacteria even in the absence of antimicrobial pressure. There is also evidence that animal gut microbiota could be colonized by resistant strains from the farm environment or from other animals by direct contact or by consumption of contaminated feed, air or water (Sayah et al., 2005; Novais et al., 2013). In our study, similar resistance profiles among E. coli isolates from farm environment and calves at birth and at 6 wk of age were detected, and in most of cases $E$. coli isolates exhibited resistance to both DO and E. In the present study, some calves were excluded from the study because of antimicrobial therapy, but they remained in contact with the other calves. Therefore, they could also be a focus of horizontal resistance transfer for the nontreated calves sampled herein. Then, the effect of calf-housing system (individual vs. group) on antimicrobial resistance in fecal $E$. coli was also evaluated in a model considering the housing system (i.e., calves grouped before or after $42 \mathrm{~d}$ of age), the feeding regimen, and their interaction as fixed effects. Contrary to the expected results, this analysis indicated that calves that were raised individually had a greater percentage of resistant bacteria to $\mathrm{CT}$ compared with calves that were grouped before $42 \mathrm{~d}$ of age. No differences were observed for the other antimicrobials analyzed.
Escherichia coli isolates from calves sampled at birth also exhibited high levels of resistance not only to DO and E, but also to FFC and S. However, resistance to FFC was not detected and resistance against $\mathrm{S}$ was low in E. coli isolates from CA. In dairy cattle, it has been suggested that the transmission of resistant bacteria could also occur from cow to calf (Watson et al., 2012), but similarities between resistance patterns of $E$. coli isolates from calves at birth and those found in their dams were not detected herein. It is possible that due to the relatively low paired samples collected from calves and their environment and from calves and their respective dams at birth, this potential association cannot be demonstrated in the current study.

Based on the assumption that resistance mechanisms impose an additional cost to bacteria (Melnyk et al., 2015), a reduction of resistance incidence in older calves not exposed to antimicrobials was expected. Others authors (Hoyle et al., 2004a,b; Berge et al., 2005) have also indicated an inverse relationship between the occurrence of resistant fecal E. coli and age. However, the prevalence of resistant $E$. coli in calves sampled at $6 \mathrm{wk}$ increased at $1 \mathrm{yr}$ of age for $\mathrm{E}, \mathrm{FFC}$, and $\mathrm{S}$. These findings are also in contrast with Khachatryan et al. (2004) who reported a fitness advantage of resistant $E$. coli in the calf enteric environment, but not in older animals.

Regarding the presence of resistant bacteria in the respiratory tract, the isolation rate of $P$. multocida from calves in the present study (36.5\% at 6 wk of age, and $1.5 \%$ at 1 yr of age) was in the range reported elsewhere. Catry et al. (2006) reported $57.4 \%$ P. multocida isolates from 61 healthy calves, whereas Allen et al. (1991) reported a prevalence rate of only $0.5 \%$ of 971. Bacteria of the genera Pasteurella are considered opportunistic pathogens and their detection has been associated with respiratory disease in cattle (Angen et al., 2009; El Garch et al., 2016). In this study, nasal swabs were collected from apparently healthy calves; thus, this would likely be the reason for the low rate of P. multocida isolation herein. Regarding WM effects on nasal microbiota, an increase of resistant $P$. multocida isolates might have been expected because of a direct contact of nasal mucosa with WM or through antimicrobial residues absorbed in the intestine. However, an increase in the prevalence of resistant $P$. multocida was only observed for CT in calves fed WM and this antimicrobial was only used in one of the MR farms (Table 2 ) to treat both respiratory and digestive infections in calves. Several mechanism of CT resistance have been described: mediated by the addition of modifying proteins to the lipid A moiety of lipopolysaccharide (Yan et al., 2007), involving mutations (Xiao et al., 2015), or plasmid-mediated (Liu et al., 2016). In this context, 
any of these resistance mechanisms could explain the CT resistance observed herein.

Similar to fecal samples, high levels of P. multocida resistant to $\mathrm{S}$ were found on all study farms. Kehrenberg et al. (2001) observed that strA and sulII, genes conferring resistance to $\mathrm{S}$ and sulfonamides, respectively, were found to be genetically linked on different types of plasmids in bacteria of the genera Pasteurella and Mannheimia. On the other hand, Guerra et al. (2003) found gene cassettes conferring resistance to S/ spectinomycin (aadA1a, aadA2, and aadA7) in all E. coli isolates from animals carrying class 1 integrons. Resistance to $\mathrm{S}$ can also arise through mutations in the $16 \mathrm{~S}$ rRNA or ribosomal proteins (O'Connor et al., 1991), but most of the mechanisms of resistance to aminoglycosides are mediated by specific aminoglycoside-modifying enzymes encoded by genes that have been found in mobile genetic elements, such as aadA and strA genes. This could be one explanation for the remarkably wide occurrence of aminoglycoside resistances observed herein (Sandvang and Aarestrup, 2000; Ramirez and Tolmasky, 2010).

In the present study, feeding WM to calves increased the presence of bacteria with antimicrobial resistance. However, the antimicrobial concentration at which these resistances appeared could not be determined because dairy farms used their own WM (different types and concentration of antimicrobials used, and different amount of WM or MR offered), and the exposure of calves to WM and MR differed among farms. Because of this diversity of the intrinsic factors among farms, farm was included in the statistical model as a random effect.

The lack of treatment interaction with calf age at 1 yr suggests that feeding WM has a time-limited effect on selecting bacteria with antimicrobial resistances. However, because animals selected at birth were not necessarily sampled at $1 \mathrm{yr}$, it was not possible to properly compare the bacteria antimicrobial pattern at birth and 1 yr later when the WM pressure was no longer present.

High levels of resistance to DO and E were observed from environmental and fecal samples of calves fed both types of milk, suggesting an environmental contamination with resistant determinants that could be transferred to calves. However, this hypothesis could not be corroborated herein because resistance was not determined genotypically.

Hence, based on the above limitation of the present study (different WM sources, different sampling periods, different use of antimicrobial and calf management), new experimental approaches under the same conditions and the incorporation of culture-independent techniques to analyze antimicrobial resistance may contribute to a better understanding of the effects of feeding calves WM.

\section{CONCLUSIONS}

Feeding calves WM fosters the presence of resistant bacteria in the gut and nasal microbiota of calves, with these changes being more evident in the gastrointestinal tract. However, high levels of resistance to erythromycin, doxycycline, and streptomycin among E. coli and to streptomycin among P. multocida isolates from calves that never received an antimicrobial therapy and were fed either MR or WM were also observed. This suggests that, in addition to the type of milk offered to calves, other factors are involved in the presence of resistant bacteria in calves' gut and nasal microbiota, such as horizontal transfer of resistant bacteria from the environment or by direct contact to other animals.

\section{ACKNOWLEDGMENTS}

The authors gratefully acknowledge the excellent technical assistance of Francesc Fàbregas and Alba Ferrer at the IRTA laboratory (Caldes De Montbui, Spain). Furthermore, the authors thank each of the dairy owners and farm personnel for providing the herd and their invaluable contributions, particularly their entry of all information needed for the project.

\section{REFERENCES}

Allen, J. W., L. Viel, K. G. Bateman, S. Rosendal, P. E. Shewen, and P. Physick-Sheard. 1991. The microbial flora of the respiratory tract in feedlot calves: Associations between nasopharyngeal and bronchoalveolar lavage cultures. Can. J. Vet. Res. 55:341-346.

Angen, Ø., J. Thomsen, L. E. Larsen, J. Larsen, B. Kokotovic, P. M. H. Heegaard, and J. M. D. Enemark. 2009. Respiratory disease in calves: Microbiological investigations on trans-tracheally aspirated bronchoalveolar fluid and acute phase protein response. Vet. Microbiol. 137:165-171.

Aust, V., K. Knappstein, H. J. Kunz, H. Kaspar, J. Wallmann, and M. Kaske. 2013. Feeding untreated and pasteurized waste milk and bulk milk to calves: Effects on calf performance, health status and antibiotic resistance of faecal bacteria. J. Anim. Physiol. Anim. Nutr. (Berl.) 97:1091-1103.

Benedict, K. M., S. P. Gow, T. A. Mcallister, C. W. Booker, S. J. Hannon, S. L. Checkley, N. R. Noyes, and P. S. Morley. 2015. Antimicrobial resistance in Escherichia coli recovered from feedlot cattle and associations with antimicrobial use. PLoS One 10:e0143995.

Berge, A. C. B., E. R. Atwill, and W. M. Sischo. 2005. Animal and farm influences on the dynamics of antibiotic resistance in faecal Escherichia coli in young dairy calves. Prev. Vet. Med. 69:25-38.

Berge, A. C. B., D. A. Moore, and W. M. Sischo. 2006. Field trial evaluating the influence of prophylactic and therapeutic antimicrobial administration on antimicrobial resistance of fecal Escherichia coli in dairy calves. Appl. Environ. Microbiol. 72:3872-3878.

Bilandžić, N., B. S. Kolanović, I. Varenina, and Z. Jurković. 2011. Concentrations of veterinary drug residues in milk from individual farms in Croatia. Mljekarstvo 61:260-267.

Bischoff, K. M., D. G. White, M. E. Hume, T. L. Poole, and D. J. Nisbet. 2005. The chloramphenicol resistance gene $\mathrm{cmlA}$ is dissemi- 
nated on transferable plasmids that confer multiple-drug resistance in swine Escherichia coli. FEMS Microbiol. Lett. 243:285-291.

Brunton, L. A., D. Duncan, N. G. Coldham, L. C. Snow, and J. R. Jones. 2012. A survey of antimicrobial usage on dairy farms and waste milk feeding practices in England and Wales. Vet. Rec. 171:296-302.

Catry, B., A. Decostere, S. Schwarz, C. Kehrenberg, A. de Kruif, and F. Haesebrouck. 2006. Detection of tetracycline-resistant and susceptible Pasteurellaceae in the nasopharynx of loose group-housed calves. Vet. Res. Commun. 30:707-715.

Clinical Laboratory Standards Institute (CLSI). 2002. Performance standards for antimicrobial disk and dilution susceptibility tests for bacteria isolated from animals; approved standard. NCCLS M31-A2. 2nd ed. CLSI (formerly the National Committee for Clinical Laboratory Standards), Wayne, PA.

Clinical and Laboratory Standards Institute (CLSI). 2008. Performance standards for antimicrobial susceptibility testing; 18th Informational Supplement M100-S18. CLSI. Wayne, PA.

Clinical and Laboratory Standards Institute (CLSI). 2013. Performance standards for antimicrobial disk and dilution susceptibility tests for bacteria isolated from animals; approved standard. VET01-A4. 4th ed. CLSI. Wayne, PA.

Clinical and Laboratory Standards Institute (CLSI). 2014. Performance standards for antimicrobial susceptibility testing; 24th Informational Supplement M100-S24. CLSI. Wayne, PA.

Cloeckaert, A., S. Baucheron, G. Flaujac, S. Schwarz, C. Kehrenberg, J. Martel, and E. Chaslus-dancla. 2000. Plasmid-mediated florfenicol resistance encoded by the floR gene in Escherichia coli isolated from cattle. Antimicrob. Agents Chemother. 44:2858-2860.

Dantzig, A. H. 1997. Oral absorption of $\beta$-lactams by intestinal peptide transport proteins. Adv. Drug Deliv. Rev. 23:63-76.

De Briyne, N., J. Atkinson, S. P. Borriello, and L. Pokludova. 2014. Antibiotics used most commonly to treat animals in Europe. Vet. Rec. 175:325-332.

Doublet, B., S. Schwarz, E. Nußbeck, S. Baucheron, J.-L. Martel, E. Chaslus-Dancla, and A. Cloeckaert. 2002. Molecular analysis of chromosomally florfenicol-resistant Escherichia coli isolates from France and Germany. J. Antimicrob. Chemother. 49:49-54.

Duse, A., K. P. Waller, U. Emanuelson, H. E. Unnerstad, Y. Persson, and B. Bengtsson. 2013. Farming practices in Sweden related to feeding milk and colostrum from cows treated with antimicrobials to dairy calves. Acta Vet. Scand. 55:49-57.

El Garch, F. E., A. De Jong, S. Simjee, H. Moyaert, U. Klein, C. Ludwig, H. Marion, S. Haag-Diergarten, A. Richard-Mazet, V. Thomas, and E. Siegwart. 2016. Monitoring of antimicrobial susceptibility of respiratory tract pathogens isolated from diseased cattle and pigs across Europe, 2009-2012: VetPath results. Vet. Microbiol. 194:11-22.

Galani, I., F. Kontopidou, M. Souli, P. Rekatsina, E. Koratzanis, J. Deliolanis, and H. Giamarellou. 2008. Colistin susceptibility testing by Etest and disk diffusion methods. Int. J. Antimicrob. Agents 31:434-439.

Gow, S. P., C. L. Waldner, J. Harel, and P. Boerlin. 2008. Associations between antimicrobial resistance genes in fecal generic Escherichia coli isolates from cow-calf herds in western Canada. Appl. Environ. Microbiol. 74:3658-3666.

Guerra, B., E. Junker, A. Schroeter, B. Malorny, S. Lehmann, and R. Helmuth. 2003. Phenotypic and genotypic characterization of antimicrobial resistance in German Escherichia coli isolates from cattle, swine and poultry. J. Antimicrob. Chemother. 52:489-492.

Gullberg, E., S. Cao, O. G. Berg, C. Ilbäck, L. Sandegren, D. Hughes, and D. I. Andersson. 2011. Selection of resistant bacteria at very low antibiotic concentrations. PLoS Pathog. 7:e1002158.

Hendriksen, R. S., D. J. Mevius, A. Schroeter, C. Teale, D. Meunier, P. Butaye, A. Franco, A. Utinane, A. Amado, M. Moreno, C. Greko, K. Stärk, C. Berghold, A.-L. Myllyniemi, D. Wasyl, M. Sunde, and F. M. Aarestrup. 2008. Prevalence of antimicrobial resistance among bacterial pathogens isolated from cattle in different European countries: 2002-2004. Acta Vet. Scand. 50:28-38.

Hoyle, D. V., H. I. Knight, D. J. Shaw, K. Hillman, M. C. Pearce, J. C. Low, G. J. Gunn, and M. E. J. Woolhouse. 2004a. Acquisition and epidemiology of antibiotic-resistant Escherichia coli in a cohort of newborn calves. J. Antimicrob. Chemother. 53:867-871.

Hoyle, D. V., D. J. Shaw, H. I. Knight, H. C. Davison, M. C. Pearce, J. C. Low, G. J. Gunn, and M. E. J. Woolhouse. 2004b. Age-related decline in carriage of ampicillin-resistant Escherichia coli in young calves. Appl. Environ. Microbiol. 70:6927-6930.

Juck, D., J. Ingram, M. Prévost, J. Coallier, and C. Greer. 1996. Nested PCR protocol for the rapid detection of Escherichia coli in potable water. Can. J. Microbiol. 42:862-866.

Kar, D., S. Bandyopadhyay, D. Bhattacharyya, I. Samanta, A. Mahanti, P. K. Nanda, B. Mondal, P. Dandapat, A. K. Das, T. K. Dutta, S. Bandyopadhyay, and R. K. Singh. 2015. Molecular and phylogenetic characterization of multidrug resistant extended spectrum beta-lactamase producing Escherichia coli isolated from poultry and cattle in Odisha, India. Infect. Genet. Evol. 29:82-90.

Kehrenberg, C., G. Schulze-Tanzil, J. L. Martel, E. Chaslus-Dancla, and S. Schwarz. 2001. Antimicrobial resistance in Pasteurella and Mannheimia: Epidemiology and genetic basis. Vet. Res. 32:323339

Kerr, D. E., and O. Wellnitz. 2003. Mammary expression of new genes to combat mastitis. J. Anim. Sci. 81:38-47.

Khachatryan, A. R., D. D. Hancock, T. E. Besser, and D. R. Call. 2004. Role of calf-adapted Escherichia coli in maintenance of antimicrobial drug resistance in dairy calves. Appl. Environ. Microbiol. 70:752-757.

Langford, F. M., D. M. Weary, and L. Fisher. 2003. Antibiotic resistance in gut bacteria from dairy calves: A dose response to the level of antibiotics fed in milk. J. Dairy Sci. 86:3963-3966.

Levison, M. E., and J. H. Levison. 2009. Pharmacokinetics and pharmacodynamics of antibacterial agents. Infect. Dis. Clin. North Am. 23:791-815.

Liu, Y. Y., Y. Wang, T. R. Walsh, L. X. Yi, R. Zhang, J. Spencer, Y. Doi, G. Tian, B. Dong, X. Huang, L. F. Yu, D. Gu, H. Ren, X. Chen, L. Lv, D. He, H. Zhou, Z. Liang, J. H. Liu, and J. Shen. 2016. Emergence of plasmid-mediated colistin resistance mechanism MCR-1 in animals and human beings in China: A microbiological and molecular biological study. Lancet Infect. Dis. $16: 161-168$.

Melnyk, A. H., A. Wong, and R. Kassen. 2015. The fitness costs of antibiotic resistance mutations. Evol. Appl. 8:273-283.

Meunier, D., E. Jouy, C. Lazizzera, B. Doublet, M. Kobisch, A. Cloeckaert, and J. Y. Madec. 2010. Plasmid-borne florfenicol and ceftiofur resistance encoded by the floR and bla $a_{\mathrm{CMY}-2}$ genes in Escherichia coli isolates from diseased cattle in France. J. Med. Microbiol. 59:467-471.

Novais, C., A. R. Freitas, E. Silveira, P. Antunes, R. Silva, T. M. Coque, and L. Peixe. 2013. Spread of multidrug-resistant Enterococcus to animals and humans: An underestimated role for the pig farm environment. J. Antimicrob. Chemother. 68:2746-2754.

O'Connor, M., E. De Stasio, and A. Dahlberg. 1991. Interaction between 16S ribosomal RNA and ribosomal protein S12: Differential effects of paromomycin and streptomycin. Biochimie 73:1493-1500.

Pereira, R. V., J. D. Siler, R. C. Bicalho, and L. D. Warnick. 2014a. In vivo selection of resistant $E$. coli after ingestion of milk with added drug residues. PLoS One 9:e115223.

Pereira, R. V., J. D. Siler, R. C. Bicalho, and L. D. Warnick. 2014b. Multiresidue screening of milk withheld for sale at dairy farms in central New York State. J. Dairy Sci. 97:1513-1519.

Philippon, A., G. Arlet, and G. A. Jacoby. 2002. Plasmid-determined AmpC-type Beta-lactamases. Antimicrob. Agents Chemother. 46:1-11.

Queenan, A. M., and K. Bush. 2007. Carbapenemases: The versatile B-lactamases. Clin. Microbiol. Rev. 20:440-458.

Ramirez, M. S., and M. E. Tolmasky. 2010. Aminoglycoside modifying enzymes. Drug Resist. Updat. 13:151-171.

Sandvang, D., and F. M. Aarestrup. 2000. Characterization of aminoglycoside resistance genes and class 1 integrons in porcine and bovine gentamicin-resistant Escherichia coli. Microb. Drug Resist. 6:19-27.

Sayah, R. S., J. B. Kaneene, Y. Johnson, and R. Miller. 2005. Patterns of antimicrobial resistance observed in Escherichia coli isolates 
obtained from domestic- and wild-animal fecal samples, human septage, and surface water. Appl. Environ. Microbiol. 71:13941404.

Shaikh, S., J. Fatima, S. Shakil, S. Mohd, D. Rizvi, and M. A. Kamal. 2015. Antibiotic resistance and extended spectrum beta-lactamases: Types, epidemiology and treatment. Saudi J. Biol. Sci. 22:90-101.

Shoemaker, N. B., H. Vlamakis, K. Hayes, and A. A. Salyers. 2001. Evidence for extensive resistance gene transfer among Bacteroides spp. and among Bacteroides and other genera in the human colon. Appl. Environ. Microbiol. 67:561-568.

Stürenburg, E., and D. Mack. 2003. Extended-spectrum $\beta$-lactamases: Implications for the clinical microbiology laboratory, therapy, and infection control. J. Infect. 47:273-295.

Townsend, K. M., A. J. Frost, C. W. Lee, J. M. Papadimitriou, and H. J. S. Dawkins. 1998. Development of PCR assays for speciesand type-specific identification of Pasteurella multocida isolates. J. Clin. Microbiol. 36:1096-1100.

Watson, E., S. Jeckel, L. Snow, R. Stubbs, C. Teale, H. Wearing, R. Horton, M. Toszeghy, O. Tearne, J. Ellis-Iversen, and N. Coldham. 2012. Epidemiology of extended spectrum beta-lactamase E. coli (CTX-M-15) on a commercial dairy farm. Vet. Microbiol. 154:339-346.

Wegener, H. C. 2003. Antibiotics in animal feed and their role in resistance development. Curr. Opin. Microbiol. 6:439-445.

White, D. G., C. Hudson, J. J. Maurer, S. Ayers, S. Zhao, M. D. Lee, L. Bolton, T. Foley, and J. Sherwood. 2000. Characterization of chloramphenicol and florfenicol resistance in Escherichia coli associated with bovine diarrhea. J. Clin. Microbiol. 38:4593-4598.

Winokur, P. L., D. L. Vonstein, L. J. Hoffman, E. K. Uhlenhopp, and G. V. Doern. 2001. Evidence for transfer of CMY-2 AmpC $\beta$-Lactamase plasmids between Escherichia coli and Salmonella isolates from food animals and humans. Antimicrob. Agents Chemother. 45:2716-2722.

Wray, C., S. Furniss, and C. L. Benham. 1990. Feeding antibiotic-contaminated waste milk to calves- Effects on physical performance and antibiotic sensitivity of gut flora. Br. Vet. J. 146:80-87.

Xiao, K., Q. Liu, X. Liu, Y. Hu, X. Zhao, and Q. Kong. 2015. Identification of the avian Pasteurella multocida phop gene and evaluation of the effects of phop deletion on virulence and immunogenicity. Int. J. Mol. Sci. 17:1-15.

Yan, A., Z. Guan, and C. R. H. Raetz. 2007. An undecaprenyl phosphate-aminoarabinose flippase required for polymyxin resistance in Escherichia coli. J. Biol. Chem. 282:36077-36089.

Yates, C. M., M. C. Pearce, M. E. J. Woolhouse, and S. G. B. Amyes. 2004. High frequency transfer and horizontal spread of apramycin resistance in calf faecal Escherichia coli. J. Antimicrob. Chemother. 54:534-537.

Yoder, P. S., N. R. St-Pierre, and W. P. Weiss. 2014. A statistical filtering procedure to improve the accuracy of estimating population parameters in feed composition databases. J. Dairy Sci. 97:5645-5656. 